\title{
Influence of a subinhibitory concentration of vancomycin on the in vitro expression of virulence-related genes in the vancomycin-resistant Enterococcus faecalis
}

\author{
Tiane Martin de Moura ${ }^{[1]}$, Fabrício Souza Campos[ ${ }^{[2]}$, Juliana Caierão ${ }^{[1]}$, \\ Ana Claudia Franco ${ }^{[2]}$, Paulo Michel Roehe ${ }^{[2]}$, Pedro Alves d'Azevedo ${ }^{[1]}$, \\ Jeverson Frazzon ${ }^{[3]}$ and Ana Paula Guedes Frazzon ${ }^{[2]}$
}

[1]. Departamento Ciências Básicas da Saúde, Universidade Federal de Ciências da Saúde de Porto Alegre, Porto Alegre, Rio Grande do Sul, Brazil. [2]. Departamento de Microbiologia, Parasitologia e Imunologia, Universidade Federal do Rio Grande do Sul, Porto Alegre, Rio Grande do Sul, Brazil. [3]. Instituto de Ciência e Tecnologia de Alimentos, Universidade Federal do Rio Grande do Sul, Porto Alegre, Rio Grande do Sul, Brazil.

\begin{abstract}
Introduction: Exposure to subinhibitory concentrations (SICs) of antimicrobials may alter the bacterial transcriptome. Methods: Here, we evaluated the expression of nine virulence-related genes in vancomycin-resistant enterococci (VRE) urinary tract infection isolates grown at SICs of vancomycin. Results: A Subinhibitory concentrations of vancomycin interferes with gene modulation, but does not affect the phenotype of a VRE strain in vitro. Conclusions: Subinhibitory concentrations of vancomycin may regulate the expression of virulence factors in vivo or contribute to the selection of vancomycin-resistant strains.
\end{abstract}

Keywords: VRE. Biofilm. Expression.

Enterococcus faecalis, an important microorganism that causes healthcare-associated infections, is part of the normal human microbiota. Enterococcus faecalis is the fifth most common pathogen that causes catheter-associated urinary tract infections (UTIs) ${ }^{(1)}$. Several different virulence factors have been reported in clinical strains of E. faecalis, including biofilm formation and the expression of surface adhesion components. The ability of $E$. faecalis to adhere to medical devices such as ureteral stents and catheters and to develop biofilms is likely associated with its pathogenicity ${ }^{(2)}$.

The biofilm on plastic (BOP) operon is essential for biofilm formation ${ }^{(3)}$. Adhesion of collagen of enterococci (ACE) and aggregation substance (ASC10) are proteins that are also crucial for biofilm production ${ }^{(4)}(5)$. The sulfur assimilation system (SUF) is responsible for iron-sulfur cluster biogenesis and is essential for cellular survival, particularly during aerobic growth or oxidative stress. The SUF operon, which is associated with virulence, is controlled by the regulatory protein sensor for oxidative stress (OxyR) and the ferric-uptake regulator (FUR) ${ }^{(6)}$.

Vancomycin-resistant enterococci (VRE) have emerged as important nosocomial pathogens worldwide. The VanA

Corresponding author: Dra. Tiane Martin de Moura. Depto. Ciências Básicas da Saúde/UFCSPA. Rua Sarmento Leite 245, sala 204, 90050-170 Porto Alegre, Rio Grande do Sul, Brasil.

Phone: 5551 3303-8825

e-mail: tianedemoura@gmail.com

Received 19 January 2015

Accepted 18 May 2015 phenotype (associated with vanA) is responsible for high levels of resistance to vancomycin ${ }^{(7)}$. The clinical use of vancomycin is a subject of major concern, because continuous monitoring is required to maintain a concentration of approximately $15 \mathrm{mg} / \mathrm{L}$ of the drug in patients' sera. Occasionally, because of the complex pharmacokinetics of vancomycin, this value is not achieved and patients are subjected to subinhibitory concentrations (SICs) of the drug. Consequently, frequent incidences of vancomycin failure and poor outcomes have been observed recently. Under these conditions, there is a high probability of selecting resistant or heteroresistant isolates ${ }^{(8)}$.

Exposure to SICs of antimicrobials may alter the transcriptomic and phenotypic responses of pathogenic bacteria $^{(9)(10)}$; however, the effect of SICs of antimicrobials on virulence remains unclear. The aim of this study was to evaluate the expression of virulence-related genes in an E. faecalis VRE strain isolated from a urinary tract infection and grown under SICs of vancomycin. The bacterial strains used in this study are listed in Table 1. The growth capacities of four E. faecalis strains were examined, and the strain with the highest optical density (OD) value of biofilm formation was selected for transcriptional profiling. All the E. faecalis strains were streaked onto Tryptic Soy Broth (TSB) agar and incubated at $37^{\circ} \mathrm{C}$ overnight (ON). Four individual colonies were used to inoculate the same tube of $5 \mathrm{~mL}$ of $2 \times$ YT broth ( $1 \%$ yeast extract, $1.6 \%$ tryptone, and $1 \% \mathrm{NaCl}$ ) and grown $\mathrm{ON}$ at $37^{\circ} \mathrm{C}$. Urine from six healthy men and women volunteers with no history of UTIs or antibiotic use in the last 6 months was collected, pooled, centrifuged, sterilized twice by filtration, and added to $2 \times$ YT broth to make 
TABLE 1 - Bacterial strains used in this study.

\begin{tabular}{|c|c|c|c|c|c|}
\hline \multirow{2}{*}{ Strain } & \multirow{2}{*}{ Source } & \multirow{2}{*}{ Isolation site/year } & \multicolumn{2}{|c|}{ Molecular identification } & \multirow{2}{*}{$\frac{\text { Resistance characteristics }}{\text { Van MIC }^{c}}$} \\
\hline & & & species $^{a}$ & $\operatorname{van} A^{\mathrm{b}}$ & \\
\hline UTI-1950 & $\begin{array}{l}\text { Hospitalized patient } \\
\text { PSC-PoA }\end{array}$ & urine/2001 & E. faecalis & positive & $384 \mu \mathrm{g} / \mathrm{mL}$ \\
\hline UTI-1953 & $\begin{array}{c}\text { Hospitalized patient } \\
\text { PSC-PoA }\end{array}$ & urine/2001 & E. faecalis & positive & $384 \mu \mathrm{g} / \mathrm{mL}$ \\
\hline UTI-2389 & $\begin{array}{c}\text { Hospitalized patient } \\
\text { PSC-PoA }\end{array}$ & urine/2002 & E. faecalis & positive & $384 \mu \mathrm{g} / \mathrm{mL}$ \\
\hline
\end{tabular}

Van: vancomycin; MIC: minimal inhibitory concentration; UTI: urinary tract infections; PSC: Pronto Socorro Central; PoA: Porto Alegre;

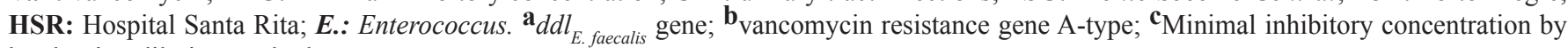
broth microdilution method.

nine different sample concentrations: $50 \%, 25 \%, 12 \%, 10 \%$, $8 \%, 6 \%, 4 \%$, and $2 \%(2 \times \mathrm{YT}-\mathrm{U} 50-2 \times \mathrm{YT}-\mathrm{U} 2)$ and pure urine $(100 \%)$. The positive control contained only $2 \times$ YT broth. The concentration of vancomycin chosen for the assays $(64 \mu \mathrm{g} / \mathrm{mL})$ was based on the range $64-1,000 \mu \mathrm{g} / \mathrm{mL}$, which has been shown to produce high levels of resistance ${ }^{(7)}$.

All the strains were used to inoculate plates containing $2 \times \mathrm{YT}$ agar and incubated at $35^{\circ} \mathrm{C} \mathrm{ON}$. All the isolates were then evaluated for their ability to form biofilms in ten different media: $2 \times$ YT broth (control), eight different concentrations of urine (2-50\%) and 100\% urine under two conditions - drug-free condition (DFC) and vancomycin condition (VC) - by using the crystal violet assay ${ }^{(11)}$. Four to eight colonies were diluted in $0.9 \%$ sterile saline $(\mathrm{w} / \mathrm{v})$ until the turbidity matched that of a $0.5 \mathrm{McF}$ arland standard [approximately $1 \times 10^{8}$ colonyforming units per milliliter (CFU/ml)]. Eight wells of a 96-well flat-bottomed microplate were filled with $180 \mu \mathrm{L}$ of each media type and $20 \mu \mathrm{L}$ of bacterial suspension. The optical density (OD) was measured at $492 \mathrm{~nm}$ in a spectrophotometer, and the OD of each strain was determined by comparing the arithmetic mean of the absorbance of the wells with the mean absorbance of the negative controls. The strains were categorized on the basis of their ODs into the following groups: non-adherent, weak biofilm producers, moderate biofilm producers, and strong biofilm producers $^{(11)}$. Non-inoculated $2 \times$ YT wells were used as negative controls, and Staphylococcus epidermidis ATCC 35984 was used as a positive control. The $\mathrm{OD}_{492}$ of each well was measured; all tests were carried out in triplicate. The effects of the different media on bacterial growth and biofilm formation were evaluated using the paired $t$-test (level of significance $=0.05$ ).

Eleven genes (vanA, bopA, bopB, bopC, bopD, ace, asc10, fur, oxyR, tuf, and 23S) were analyzed by quantitative polymerase chain reaction (qPCR). The oligonucleotides used in this study are listed in Table 2. Only one strain, exhibiting the maximum OD value, was selected for transcriptional profiling. The qPCR analyses were performed at least three times. Fivehundred microliters of ON culture were inoculated in $49.5 \mathrm{~mL}$ of $2 \times$ YT-U10 in the absence (DFC) and presence (VC) of SICs of vancomycin. Aliquots were collected and extracted at the early-exponential phase $\left(\mathrm{OD}_{650} 0.3\right)$, total ribonucleic acid (RNA) was purified and quantified, and cDNAs were synthesized $^{(12)}$. Quantitative PCRs were performed as uniplex reactions in final volumes of $20 \mu \mathrm{L}$ with $100 \mathrm{pg}$ of cDNA using the Eco ${ }^{\mathrm{TM}}$ Real Time PCR System (Illumina ${ }^{\circledR}$ San Diego, USA) and universal cycling protocols.

We analyzed the effect of different concentrations of urine on biofilm formation by phenotypic characterization and on the basis of the OD values. Biofilm formation in the VRE strains was negatively influenced by the presence of pure urine at $35^{\circ} \mathrm{C}$ (no growth was observed). With urine concentrations of $2-50 \%$, all the strains showed growth $(\mathrm{OD}, 0.016 \pm 0.004)$; however, they were unable to form biofilms under both conditions. The UTI2389 isolate produced the highest OD value $(0.021 \pm 0.004)$. No significant association was observed between biofilm formation and urine concentration. These findings might be a consequence of the in vitro environment, which lacked essential components of the in vivo urinary system such as urothelial cells, glucose, mineral salts, and albumin. Moreover, it has been suggested that biological signals in human urine play an important role in modulating virulence at enterococci infection sites ${ }^{(13)}$. On the basis of our biofilm assay, all the strains could be classified as non-adherent according to both DFC and VC. Although maximal biofilm induction has been observed at a concentration of $3 / 4^{\text {th }}$ the minimal inhibitory concentration $(\mathrm{MIC})^{(10)}$, in other studies, vancomycin at concentrations $\leq 1 / 2$ the $\mathrm{MIC}^{(9)}$ had little or no effect on $S$. epidermidis biofilm formation. Thus, we concluded that the low concentration of vancomycin used in our study $\left(1 / 6^{\text {th }}\right.$ the MIC) produced no effect on biofilm formation, which is consistent with the OD values observed in our study.

On the basis of the results of our biofilm assays, we selected the UTI-2389 strain and $10 \%$ urine $(2 \times$ YT-U10) for subsequent qPCR analysis. The relative quantification of the messenger ribonucleic acid (mRNA) concentrations is 
TABLE 2 - List of genes and oligonucleotides used for qPCR analyses.

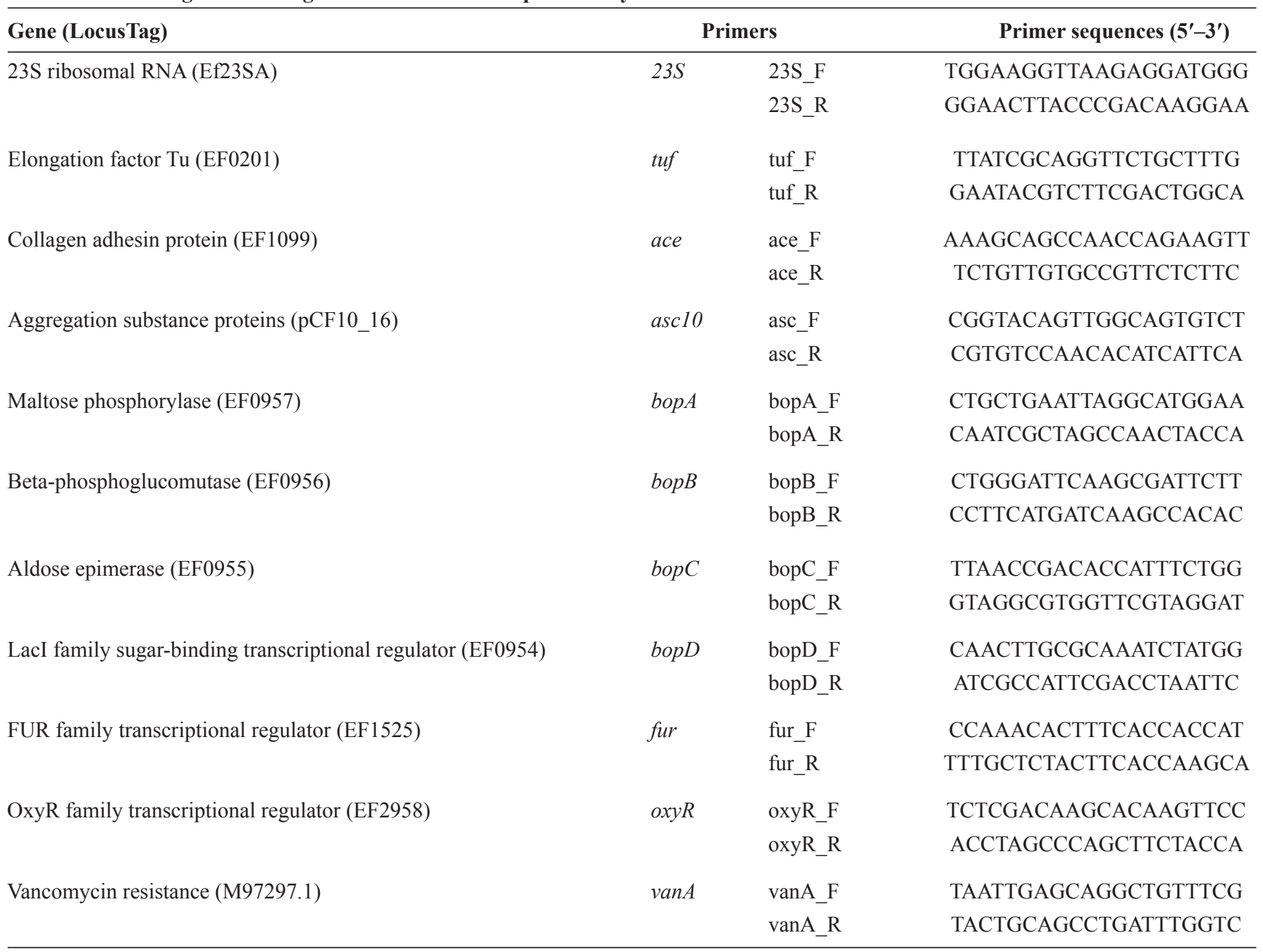

qPCR: quantitative polymerase chain reaction; RNA: ribonucleic acid; LacI: lactose; FUR: ferric-uptake regulator; OxyR: oxidative stress regulator; tuf: elongation factor Tu; ace: adhesion of collagen of enterococci; asc10: aggregation substance; bop: biofilm on plastic; vanA: vancomycin resistance gene A-type; F: primer sense, R: primer antisense. The oligonucleotides were designed using bioinformatics tools (https:/www.genscript.com/ssl-bin/app/primer)

shown in Table 3. Significant upregulation of vanA, which is vancomycin-inducible ${ }^{(7)}$, was detected under the $\mathrm{VC}$ condition. We used this result to investigate the up/downregulation of the other selected genes. Genes of the bop operon were partially regulated in the presence of SICs of vancomycin. Treatment with SICs of vancomycin significantly upregulated bopA and bopC and slightly downregulated bopB and bopD. Similar partial regulation of the bop operon in a VRE strain has been reported previously ${ }^{(14)}$ under growth conditions lacking antimicrobial agents, which suggests that this operon behaves erratically, even under normal growth conditions. Under the conditions tested, the expression of ace was significantly downregulated. However, in a previous study, ace expression was scarcely detected ${ }^{(15)}$ in cells at the mid- and late exponential phases and was not detectable in cells during the stationary phase, suggesting that ace transcription is very low under standard in vitro growth conditions. The expression of acs 10 was induced slightly by SICs of vancomycin. ASC10 is expressed in the presence of an intercellular signal cCF10, which is a peptide pheromone ${ }^{(4)}$. Moreover, antibiotics can act as signaling molecules; therefore, vancomycin could have induced asc10 expression. We also detected minor downregulation of $\operatorname{oxy} R$. During intracellular oxidative stress, the $E$. faecalis SUF system is induced by OxyR and requires the expression of the integration host factor ${ }^{(6)}$. In this in vitro study, no hydrogen peroxide production was observed, which may explain the lack of stimulation of $\operatorname{oxy} R$. In contrast to $\operatorname{oxy} R$, the expression of the ferric uptake regulator fur was significantly upregulated in response to the stress caused by vancomycin, indicating that this gene is not regulated in the same manner as $\operatorname{oxy} R$. For example, fur may operate in response to a cofactor in cell homeostasis, because the SUF system is the housekeeping machinery for $E$. faecalis ${ }^{(6)}$.

In summary, we present here, for the first time, data on the behavior of VRE from a UTI strain in the presence of SICs of vancomycin. The nine genes evaluated in this study are related to virulence factors that are often present 
TABLE 3 - Delta cycle threshold $(\Delta C t)$ and ratio values of the expression of virulence related genes in a vancomycin-resistant enterococcus strain grown in $2 \times$ YT $+10 \%$ urine in absence (DFC) and presence (VC) of subinhibitory concentrations of vancomycin.

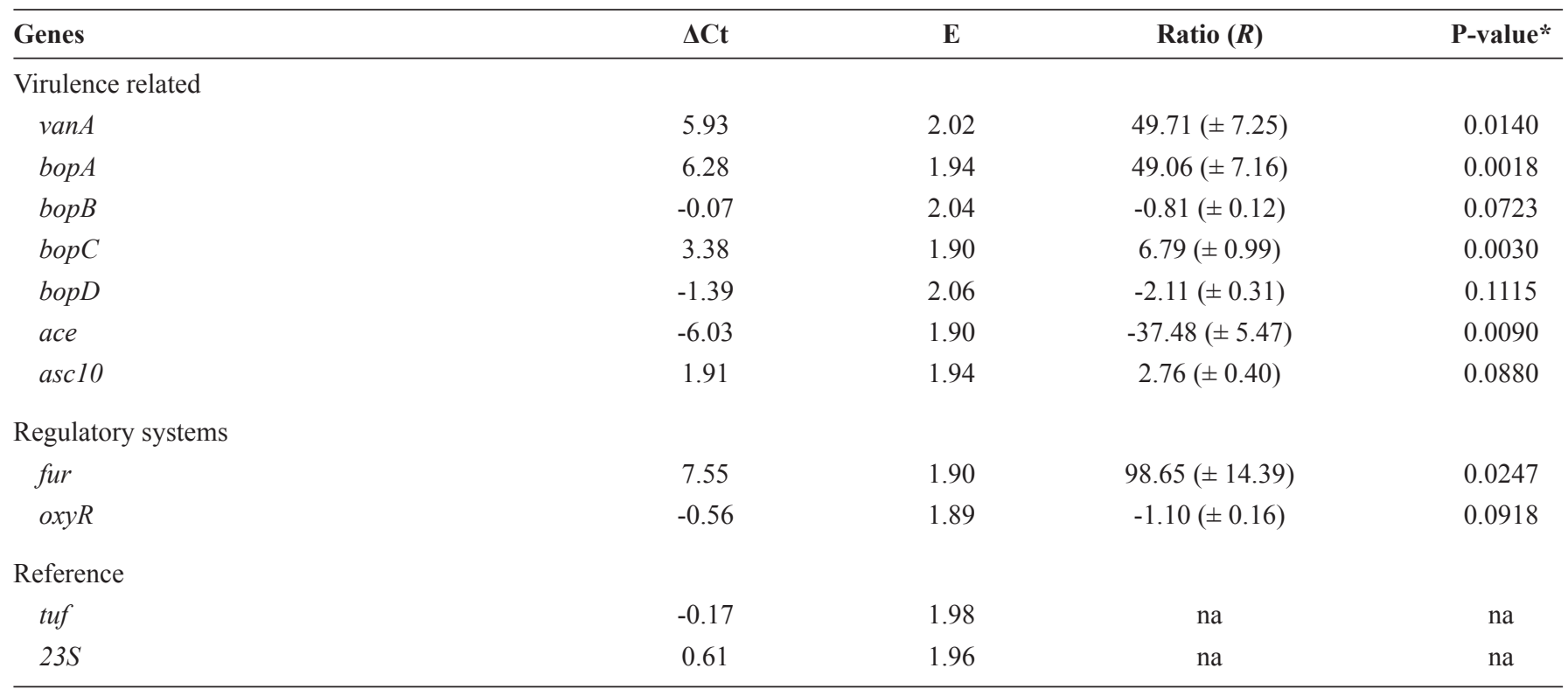

$\mathbf{\Delta C t}$ : cycle threshold variation; YT: yeast extract, tryptone and $\mathrm{NaCl}$ broth; DFC: drug-free condition; VC: vancomycin condition; $\mathbf{E}$ : efficiency of the reaction; R: the relative expression ratio is the average value of the normalized based on the reference genes; vanA: vancomycin resistance gene A-type; bop: biofilm on plastic; ace: adhesion of collagen of enterococci; asc10: aggregation substance; fur: ferric-uptake regulator; oxyR: oxidative stress regulator; tuf: elongation factor Tu; 23S ribosomal RNA; na: not applicable; *Level of significance $=0.05$.

in Enterococcus faecalis. Although there was an increase in the expression of several VRE genes in the presence of SICs of vancomycin, no differences in their phenotypes were observed in vitro. Although the data presented here are from in vitro assays, SICs of vancomycin may contribute to a similar increase in the expression of virulence factors in vivo, leading to adverse clinical outcomes and to the selection of vancomycin-resistant strains. In-vitro experiments may not effectively represent the complex in vivo conditions during enterococcal urinary tract infection. Therefore, additional in vitro and in vivo studies should be carried out to corroborate our findings. The current study is limited because the strains tested were isolated at local hospitals before the study commenced, meaning that we have no patient records detailing their treatments or clinical outcomes. Our main interest was to investigate the behavioral response of a clinical VRE strain against SICs of vancomycin. Therefore, we did not perform tests with other antimicrobials or compare our results to a reference strain.

\section{CONFLICT OF INTEREST}

The authors declare that there is no conflict of interest.

\section{REFERENCES}

1. Sievert DM, Ricks P, Edwards JR, Schneider A, Patel J, Srinivasan A, et al. National Healthcare Safety Network (NHSN) Team and Participating NHSN Facilities 2013. Antimicrobial-resistant pathogens associated with healthcare-associated infections: summary of data reported to the National Healthcare Safety Network at the Centers for Disease Control and Prevention, 20092010. Infect Control Hosp Epidemiol 2013; 34:1-14.

2. Mohamed JA, Huang DB. Biofilm formation by enterococci. J Med Microbiol 2007; 56 (Pt 12):1581-1588.

3. Hufnagel M, Koch S, Creti R, Baldassarri L, Huebner J. A putative sugar-binding transcriptional regulator in a novel gene locus in Enterococcus faecalis contributes to production of biofilm and prolonged bacteremia in mice. J Infect Dis 2004; 189:420-430.

4. Waters CM, Dunny GM. Analysis of functional domains of the Enterococcus faecalis pheromone-induced surface protein aggregation substance. J Bacteriol 2001; 183:5659-5667.

5. Chuang-Smith ON, Wells CL, Henry-Stanley MJ, Dunny GM. Acceleration of Enterococcus faecalis biofilm formation by aggregation substance expression in an ex vivo model of cardiac valve colonization. PLoS One 2010; 5:e15798.

6. Riboldi GP, de Mattos EP, Frazzon J. Biogenesis of [Fe-S] cluster in Firmicutes: an unexploited field of investigation. Antonie Van Leeuwenhoek 2013; 104:283-300.

7. Courvalin P. Vancomycin resistance in gram-positive cocci. Clin Infect Dis 2006; 42:S25-S34.

8. van Hal SJ, Fowler Jr VG. Is it time to replace vancomycin in the treatment of methicillin-resistant Staphylococcus aureus infections? Clin Infect Dis 2013; 56:1779-1788.

9. Cargill JS, Upton M. Low concentrations of vancomycin stimulate biofilm formation in some clinical isolates of Staphylococcus epidermidis. J Clin Pathol 2009; 62:1112-1116.

10. Kaplan JB, Jabbouri S, Sadovskaya I. Extracellular DNAdependent biofilm formation by Staphylococcus epidermidis RP62A in response to subminimal inhibitory concentrations of antibiotics. Res Microbiol 2011; 162:535-541. 
11. Stepanovic S, Vukovic D, Dakic I, Savic B, Svabic-Vlahovic M. A modified microtiter-plate test for quantification of staphylococcal biofilm formation. J Microbiol Methods 2000; 40:175-179.

12. Moura TM, Cassenego AP, Campos FS, Ribeiro AM, Franco $\mathrm{AC}$, d'Azevedo PA, et al. Detection of vanC1 gene transcription in vancomycin-susceptible Enterococcus faecalis. Mem Inst Oswaldo Cruz 2013; 108:453-456.

13. Shepard BD, Gilmore MS. Differential expression of virulencerelated genes in Enterococcus faecalis in response to biological cues in serum and urine. Infect Immun 2002; 70:4344-4352.
14. Vebø HC, Solheim M, Snipen L, Nes IF, Brede DA. Comparative genomic analysis of pathogenic and probiotic Enterococcus faecalis isolates, and their transcriptional responses to growth in human urine. PLoS One 2010; 5: e12489.

15. Nallapareddy SR, Murray BE. Ligand-signaled upregulation of Enterococcus faecalis ace transcription, a mechanism for modulating host-E. faecalis interaction. Infect Immun 2006; 74:4982-4989. 